Published in final edited form as:

Nat Plants. 2019 January ; 5(1): 26-33. doi:10.1038/s41477-018-0320-9.

\title{
Sensitive detection of pre-integration intermediates of LTR retrotransposons in crop plants
}

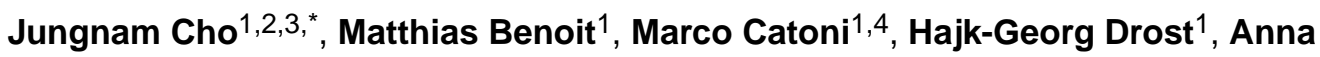 \\ Brestovitsky ${ }^{1}$, Matthijs Oosterbeek ${ }^{5,6}$, and Jerzy Paszkowski ${ }^{1, *}$
}

${ }^{1}$ The Sainsbury Laboratory, University of Cambridge, Cambridge CB2 1LR, UK ${ }^{2}$ National Key Laboratory of Plant Molecular Genetics (NKLPMG), CAS Center for Excellence in Molecular Plant Sciences, Institute of Plant Physiology and Ecology (SIPPE), 200032 Shanghai, P. R. China ${ }^{3}$ CAS-JIC Centre of Excellence for Plant and Microbial Science (CEPAMS), Chinese Academy of Sciences, 200032 Shanghai, P. R. China ${ }^{4}$ School of Biosciences, University of Birmingham, Birmingham BI5 2TT, UK ${ }^{5}$ Laboratory of Molecular Biology, Wageningen University, Wageningen 6708PB, The Netherlands

\section{Abstract}

Retrotransposons have played an important role in the evolution of host genomes1,2. Their impact is mainly deduced from the composition of DNA sequences that have been fixed over evolutionary time 2. Such studies provide important "snapshots" reflecting the historical activities of transposons but do not predict current transposition potential. We previously reported SequenceIndependent Retrotransposon Trapping (SIRT) as a method that, by identification of extrachromosomal linear DNA (ecIDNA), revealed the presence of active LTR retrotransposons in Arabidopsis3. However, SIRT cannot be applied to large and transposon-rich genomes, as found in crop plants. We have developed an alternative approach named ALE-seq (amplification of $\underline{L}$ TR of eclDNAs followed by sequencing) for such situations. ALE-seq reveals sequences of 5' LTRs of eclDNAs after two-step amplification: in vitro transcription and subsequent reverse transcription. Using ALE-seq in rice, we detected ecIDNAs for a novel Copia family LTR retrotransposon, Goon, which is activated by heat stress. Sequencing of rice accessions revealed that Go-on has preferentially accumulated in indica rice grown at higher temperatures. Furthermore, ALE-seq

Users may view, print, copy, and download text and data-mine the content in such documents, for the purposes of academic research, subject always to the full Conditions of use:http://www.nature.com/authors/editorial_policies/license.html\#terms

*Corresponding authors: Correspondence to Jungnam Cho (jungnam.cho@slcu.cam.ac.uk) and Jerzy Paszkowski (jerzy.paszkowski@ @lcu.cam.ac.uk).

${ }^{6}$ Current address: Laboratory of Nematology, Wageningen University, Wageningen 6708PB, The Netherlands

Data availability

The next generation sequencing data that support the findings of this study are available in the Sequence Read Archive (SRA) repository with the identifier SRP155920.

Code availability

The custom scripts used in this study are available in http://github.com/HajkD/ALE.

Author Contributions

J.C. and J.P. conceived the research. J.C., M.B., M.C., H.-G.D., A.B. and M.O. performed experiment. J.C., M.B., M.C. and H.-G.D. analysed data. J.C. and J.P. wrote and revised the manuscript.

Competing Interests

The authors declare that no competing interests exist. 
applied to tomato fruits identified a developmentally regulated Gypsy family of retrotransposons. A bioinformatic pipeline adapted for ALE-seq data analyses is used for the direct and referencefree annotation of new, active retroelements. This pipeline allows assessment of LTR retrotransposon activities in organisms for which genomic sequences and/or reference genomes are either unavailable or of low quality.

Chromosomal copies of activated retrotransposons containing long terminal repeats (LTRs) are transcribed by RNA polymerase II, followed by reverse transcription of transcripts to extrachromosomal linear DNAs (eclDNA); these integrate back into host chromosomes3. Because of the two obligatory template switches during reverse transcription, the newly synthetized ecIDNA is flanked by LTRs of identical sequence. Their subsequent divergence due to the accumulation of mutations correlates well with length of time since the last transposition, and thus transposon age4. However, the age of LTR retrotransposons cannot be used to predict their current transpositional potential. Moreover, predictions are further complicated by recombination events that occur with high frequency between young and old members of a retrotransposon family5; thus old family members also contribute to the formation of novel recombinant elements that insert into new chromosomal positions5. Although, retrotransposon activities can be relatively easily measured at the transcriptional level6, the presence of transcripts is a poor predictor of transpositional potential due to posttranscriptional control of this process 7,8 . In addition, direct detection of transposition by genome-wide sequencing to identify new insertions is too expensive and time-consuming to be applied as a screening method. Clearly, the development of an expeditious approach to identify active retrotransposons that predict their transposition potential would be welcomed. We previously described the SIRT strategy for Arabidopsis that led to the identification of ecIDNA of a novel retroelement and subsequent detection of new insertions3. Thus, the presence of ecIDNAs, the last pre-integration intermediate, was shown to be a good predictor of retrotransposition potential.

\section{Results}

\section{Development of ALE-seq}

Retrotransposons include a conserved sequence known as the primer binding site (PBS), where binding of the 3' end of cognate tRNA initiates the reverse transcription reaction3. Met-iCAT (Methionine tRNA-CAT anticodon) PBS was chosen for SIRT as it is the site present in the majority of annotated Arabidopsis retrotransposons3. To examine whether Met-iCAT PBS sequences are also predominant in LTR retrotransposons of other plants, we used the custom-made software LTRpred for de novo annotation of LTR retrotransposons in rice and tomato genomes (see Materials and methods). Young retroelements were selected by filtering for at least $95 \%$ identity between the two LTRs and subsequently examined for their cognate tRNAs (Supplementary Figure 1). As in Arabidopsis, around 80\% of LTR retrotransposons in the tomato genome contained Met-iCAT PBS (Supplementary Figure 1). In contrast, only $30 \%$ harboured Met-iCAT PBS in rice, and Arg-CCT (Arginine tRNA-CCT anticodon) PBS was found in 60\% of young LTR retrotransposons (Supplementary Figure 1). Nonetheless, we used Met-iCAT PBS in our initial experiments because most retrotransposons known to be active in rice callus (e.g. Tos17 and Tos19) contain Met-iCAT 
PBS. Initially, SIRT was performed on DNA extracted from rice leaves and calli; however, we did not detect ecIDNAs for Tos 17 and Tos 19 in rice tissues by this method (Supplementary Figure 2). We reasoned that the short stretch of PBS used for primer design in SIRT may have impaired PCR efficiency due to the many PBS-related sequences present in larger genomes containing a high number of retroelements, as is the case in rice.

To counter this problem, we developed an alternative method, named ALE-seq, with significantly improved selectivity and sensitivity of eclDNA detection. A crucial difference to SIRT is that ALE-seq amplification of ecIDNA is separated into two reactions: in vitro transcription and reverse transcription (Figure 1a). This decoupling of the use of the two priming sequences followed by the digestion of non-templated DNA and RNA is significantly more selective and efficient than the single PCR amplification in SIRT.

ALE-seq starts with ligation to the ends of ecIDNA of an adapter containing a T7 promoter sequence at its 5' end and subsequent in vitro transcription with T7 RNA polymerase. The synthesized RNA is then reverse transcribed using the primer that binds the transcripts at the PBS site. The adapter and the oligonucleotides priming reverse transcription are anchored with partial Illumina adapter sequences (Supplementary Table 1), which allows the amplified products to be directly deep-sequenced in a strand-specific manner. The ALE-seqsequences derived from retrotransposon ecIDNAs are predicted to contain the intact 5' LTR up to the PBS site, flanked by Illumina paired-end sequencing adapters. We used the Illumina MiSeq platform for sequencing because its long reads of $300 \mathrm{bp}$ from both ends cover the entire LTR lengths of most potentially active elements. It is worth noting that the Illumina adapters were tagged to the intact LTR DNA without fragmentation of the amplicons. This together with the long reads of MiSeq allowed us to reconstitute the complete LTR sequences, even in the absence of the reference genome sequence. The reconstituted LTRs were analysed using the alignment-based approach that complements the mapping-based approach when the reference genome is incomplete (Figure 1b).

First, we tested ALE-seq on Arabidopsis by examining heat-stressed Col-0 Arabidopsis plants9, met1-1 mutant3 and epi128, a met1-derived epigenetic recombinant inbred line. ALE-seq cleanly and precisely recovered sequences of complete LTRs for Onsen, Copia21 and Evade in samples containing their respective ecIDNA (Supplementary Figure 3)3,8,9. Due to priming of the reverse transcription reaction at PBS, the reads were explicitly mapped to the 5' but not to the 3' LTR, although the two LTRs have identical sequences. The ALE-seq reads have well-defined extremities, starting at the position marking the start of LTRs and finishing at the PBS, which is consistent with their eclDNA origin. The ends of LTRs can also be inspected for conserved sequences that would further confirm their eclDNA origin (Supplementary Figure 4). This reduced ambiguity of read mapping in ALEseq analysis, combined with the clear-cut detection of LTR ends, allows for explicit and precise assignment of ALE-seq results to active LTR retrotransposons.

Since SIRT failed to detect ecIDNAs of rice retrotransposons known to be activated in rice callus, we examined whether ALE-seq would identify their eclDNAs. As shown in Figure 1c to f, ALE-seq unambiguously detected ecIDNAs of Tos 17 and Tos 19 in rice callus, but not in leaf samples. To test whether detection of 5' LTR sequences requires the entire ALE-seq 
procedure, we performed control experiments with depleted ALE-seq reactions, for example, in the absence of enzymes for either ligation, in vitro transcription, or reverse transcription. All incomplete procedures failed to produce sequences containing 5' LTRs derived from ecIDNAs (Figure 1e and f). Taken together, the data show that ALE-seq can detect ecIDNAs of LTR retrotransposons in Arabidopsis as well as in rice with considerably greater efficiency than the SIRT method.

To examine the suitability of ALE-seq for quantitative determination of ecIDNA levels, we carried out a reconstruction experiment spiking $100 \mathrm{ng}$ of genomic DNA from rice callus with differing amounts of PCR-amplified full-length Onsen DNA from $1 \mathrm{ng}$ to $100 \mathrm{fg}$ (Figure 2a to d). The results in Figure 2a and b show that the readouts of ALE-seq for Onsen correlate well with the input amounts $\left(\mathrm{R}^{2}=0.99\right)$. The initial ALE-seq steps of ligation and in vitro transcription impinged proportionally on the input DNA, resulting in unbiased quantification of the ecIDNA and minimal quantitative distortion of the final ALE-seq data. Noticeably, the levels of Tos 17 were similar in all the spiked samples, indicating that addition of Onsen DNA did not influence the detection sensitivity of Tos 17, at least for the amounts tested (Figure $2 \mathrm{c}$ and d). Thus, ALE-seq can be used to accurately determine ecIDNA levels.

Most rice retrotransposons harbour Arg-CCT PBS (Supplementary Figure 1). We tested whether the reverse transcription reaction can be multiplexed to capture both types of retrotransposons (containing Arg-CCT or Met-iCAT PBS) and whether multiplexing of the reverse transcription primers compromises the sensitivity of the procedure. ALE-seq was performed on DNA from rice callus, testing each of the reverse transcription primers separately or as a mixture of both primers in a single reaction. As shown in Figure 2e and Supplementary Figure 5, the levels of Tos 17 recorded in the samples with both primers were similar to the Met-iCAT primer alone. Importantly, we also detected the ecIDNAs of the RIRE2 element containing Arg-CCT PBS (Figure 2f), which was known to be transpositionally active in rice callus7.

\section{Identification of Go-on retrotransposon using ALE-seq}

We next used ALE-seq to search for novel active rice retrotransposons. Since many plant retrotransposons are transcriptionally activated by abiotic stresses 9,10 , we subjected rice plants to heat stress before subjecting them to ALE-seq. In this way we identified a Copiatype retrotransposon able to synthetize ecIDNA in the heat-stressed plants (Figure 3a to c) and named this element Go-on (the Korean for 'high temperature'). The three retrotransposons with the highest eclDNA levels in heat-stress conditions all belong to the Go-on family (Figure 3b and Supplementary Figure 6). Although, ecIDNAs were detected for all three copies, Go-on3 seems to be the youngest and, thus, possibly the most active family member, containing identical LTRs and a complete ORF (Supplementary Figure 6). As depicted in Supplementary Figure 6, the 5' LTR sequences of the three Go-on copies are identical; thus the ALE-seq reads derived from Go-on3 LTR were also cross-mapped to other copies that are possibly inactive or have reduced activities. To further determine whether sequences of Go-on LTRs recovered by ALE-seq are indeed derived from Go-on3 or also from other family members, we performed an ALE-seq experiment using RT primers 
located further downstream of the PBS, including sequences specific for each Go-on family member (Supplementary Figure 6). The amplified ALE-seq products revealed that the eclDNAs produced in heat-stressed rice originated only from Go-on3 (Supplementary Figure 6). We validated the production of ecIDNAs of Go-on3 by sequencing the junction of the adapter and the 5' end of LTR (Supplementary Figure 6) and by qPCR (Supplementary Figure 7).

Next, we examined whether Go-on3 is transcriptionally activated in rice subjected to heat stress. RNA-seq and the RT-qPCR data clearly showed that Go-on is strongly activated in heat-stress conditions (Figure 3d and Supplementary Figure 7). Similar to many other retrotransposons including ONSEN of Arabidopsis9,11,12, the LTR sequence of Go-on3 contains cis-acting regulatory element such as the heat shock transcription factor HSFC1binding sequence motif (Supplementary Figure 7), which is suggestive to its heat stressmediated transcriptional activation (Figure 3d). To determine whether Go-on is also activated in indica rice, we heat-stressed plants of IR64 for three days and examined Go-on RNA and DNA levels. Similar to japonica rice, Go-on RNA and DNA accumulated markedly under heat stress (Supplementary Figure 8), suggesting that the trigger for Go-on activation is conserved in both of these evolutionarily distant rice genotypes. Analysis of the RNA-seq data from the heat-stressed rice plants revealed a poor correlation between the mRNA and ecIDNA levels of retrotransposons (Supplementary Figure 9). Given that ecIDNAs captured by ALE-seq in Arabidopsis and rice (Figure 1c to $\mathrm{f}$ and Supplementary Figure 3) are all known well for their transposition competence, this possibly agrees with the notion that the ecIDNA level is a better predictor of retrotransposition than the RNA level.

To possibly relate accumulation of Go-on copies in plant populations grown in different temperatures, we analysed the historical retrotransposition of Go-on using the genome resequencing data of rice accessions from the 3,000 Rice Genome Project13. First, we retrieved the raw sequencing data for all 388 japonica rice accessions and the same number of randomly selected sequences of indica rice accessions. Using the Transposon Insertion Finder (TIF) tool14, japonica and indica sequences were analysed for the number of Go-on copies and their genome-wide distribution. Only non-reference insertions that were absent in the reference genome were scored and the cumulative number of new insertions was plotted (Figure $3 \mathrm{e}$ to g). Figure $3 \mathrm{e}$ shows that the indica rice population grown in a warmer climate 15 accumulated significantly more Go-on copies than the japonica population. As controls, we also examined the accumulation of $\operatorname{Tos} 17$ and $\operatorname{Tos} 19$, which were not activated by heat stress in our ALE-seq profile (Figure 3a and b). Both retrotransposons showed more transposition events in japonica than in indica rice (Figure 3f, g and Supplementary Figure 10). Therefore, the copy number of Go-on in rice accessions correlated with their growth temperatures, which could possibly be related to occasional Go-on activation in elevated ambient temperatures.

\section{Identification of FIRE retrotransposon using ALE-seq}

It was reported previously that the tomato genome (Solanum lycopersicum) experiences a significant loss of DNA methylation in fruits during their maturation, which leads to transcriptional activation of retrotransposons16. However, it was not known whether these 
transcriptionally activated tomato transposons synthesise ecIDNA. It was questionable whether the ALE-seq strategy is sensitive enough to detect ecIDNA in the $~ 950 \mathrm{Mb}$ tomato genome, which is almost three times as large as $\sim 400 \mathrm{Mb}$ of rice 17 . To address these questions, ALE-seq was carried out on DNA samples from fruits at 52 days post anthesis (DPA), when the loss of DNA methylation is most pronounced16, and from leaves as a control. It is important to note that we used tomato cultivar (cv.) M82 for these experiments, as it is commonly used for genetic studies 18,19 , and that the sequence of the current tomato reference genome is based on cv. Heinz 170617. Since retrotransposon sequences and their chromosomal distributions differ largely between genomes of different varieties within the same plant species20-22, we could not use the standard mapping-based annotation of the ALE-seq results. As a consequence, we developed a reference-free and alignment-based approach that adopts the clustering of reads based on their sequence similarities (Figure 1b). Briefly, the reads from both samples were pooled and then clustered by sequence homology (See Materials and methods). The consensus of each cluster was determined and used as the reference in paired-end mapping. Subsequently, the consensus sequences were used for a BLAST search against the reference genome for the closest homologues. In this way, the BLAST search was able to map the clustered ALE-seq output to reference genome annotated retrotransposons, which are most similar to the ALE-seq recovered sequences. Applying this strategy, we identified a retroelement belonging to a Gypsy family (FIRE, Fruit-Induced RetroElement) that produces significant amounts of ecIDNA at 52 DPA during fruit ripening (Figure $4 \mathrm{a}$ and $\mathrm{b}$ ). We also determined the transcript levels of the FIRE element in leaves and 52 DPA fruit samples. As shown in Figure 4c, fruit RNA levels were enhanced twofold compared to leaves, where FIRE ecIDNA was barely detectable (Figure 4a). Finally, we found that the DNA methylation status of the FIRE element was lower in fruits than leaves in all three sequence contexts (Figure $4 \mathrm{~d}$ and $\mathrm{f}$ ). In contrast, the DNA methylation levels of sequences directly flanking FIRE were similar in leaves and fruits (Figure 4e to g).

\section{Discussion}

Recently, a novel active retrotransposon was identified in rice by sequencing extrachromosomal circular DNA (eccDNA) produced as a by-product of retrotransposition or by nuclear recombination reactions of ecIDNAs23,24. Although the method of eccDNA sequencing has certain advantages over SIRT, such as increased sensitivity and the recovery of sequences of the entire element, it also has certain limitations. For example, the method requires relatively large amounts of starting material but still shows serious limits in efficiency and indicative power for retrotransposition. The method did not detect the eccDNA of Tos 19 in rice callus, where this transposon is known to move23, however, direct comparison of both methods on the same biological samples was not performed. More importantly, eccDNAs may also be the result of genomic DNA recombination 25 and these background products may be misleading when extrapolating to the transpositional potential of a previously unknown element. In this respect, ALE-seq is a significantly improved tool that largely overcomes the above-mentioned limitations of previous methods and requires only $100 \mathrm{ng}$ of plant DNA. 
The heat-responsiveness of Go-on, the novel heat-activated Copia family retrotransposon of rice detected using ALE-seq, seems to be conferred by cis-acting DNA elements embedded in the LTR, which are similar to the heat-activated Onsen retrotransposon in Arabidopsis 11,12 . Although heat stress can induce production of mRNA and eclDNA of Onsen, its retrotransposition is tightly controlled by the small interfering RNA pathway9. Given that real-time transposition of rice retrotransposons has only been detected in epigenetic mutants 26,27 and triggered by tissue culture conditions causing vast alterations in the epigenome7,or as a result of interspecific hybridization28, an altered epigenomic status seems to be an important prerequisite for retrotransposition. In fact, we failed to detect transposed copies of Go-on in the progeny of heat-stressed rice plants. Thus, although Go-on produces ecIDNAs after heat stress, it may be mobilized only at low frequency in wild type rice due to epigenetic restriction of retrotransposition. Nevertheless, on an evolutionary scale, the higher number of new insertions of Go-on in indica rice populations grown at elevated temperatures might suggest its potential mobility.

Many retrotransposons are transcriptionally reactivated during specific developmental stages or in particular cell types29,30. In tomato, fruit pericarp exhibits a reduction in DNA methylation during ripening 16 . This is largely attributed to higher transcription of the DEMETER-LIKE2 DNA glycosylase gene31. Despite massive transcriptional reactivation of retrotransposons in tomato fruits, it has been difficult to determine whether further steps toward transposition also take place. Using ALE-seq, we identified ecIDNA that we annotated using a reference-free and alignment-based approach to a novel FIRE element. $F I R E$ has 164 copies in the reference tomato genome and in a conventional mapping-based approach the ALE-seq reads of FIRE cross-mapped to multiple copies, making it difficult to assign ecIDNA levels to particular family members (Supplementary Figure 11). Therefore, our strategy can be used in situations where sequence of the reference genome is unavailable or the mapping of reads is hindered by the high complexity and multiplicity of the retrotransposon population.

ALE-seq could also be applied to non-plant systems. For example, numerous studies in various eukaryotes, including mammals, found that retrotransposons are transcriptionally activated by certain diseases or at particular stages during embryo development 32,33 . It was also suggested that retrotransposition might be an important component of disease progression34. Given that the direct detection of retrotransposition is challenging, it would be interesting to use ALE-seq to determine whether such temporal relaxations of epigenetic transposon silencing also result in the production of the ecIDNAs, as the direct precursor of the chromosomal integration of a retrotransposon.

\section{Materials and methods}

\section{Plant materials}

Seeds of Oryza sativa ssp. japonica cv. Nipponbare and Oryza sativa ssp. indica cv. IR64 were surface-sterilized in $20 \%$ bleach for $15 \mathrm{~min}$, rinsed three times with sterile water and germinated on $1 / 2-\mathrm{MS}$ media. Rice plants were grown in $10 \mathrm{~h} \mathrm{light} / 14 \mathrm{~h}$ dark at $28^{\circ} \mathrm{C}$ and $26^{\circ} \mathrm{C}$, respectively. For heat-stress experiments, 1-week-old rice plants were transferred to a 
growth chamber at $44^{\circ} \mathrm{C}$ and $28^{\circ} \mathrm{C}$ in light and dark, respectively. Rice callus was induced by the method used for rice transformation as previously described 35 .

Tomato plants (Solanum lycopersicum cv. M82) were grown under standard greenhouse conditions ( $16 \mathrm{~h}$ supplemental lighting of $88 \mathrm{w} / \mathrm{m}^{2}$ at $25^{\circ} \mathrm{C}$ and $8 \mathrm{~h}$ at $15^{\circ} \mathrm{C}$ ). Tomato leaf tissue samples were taken from 2-month-old plants. Tomato fruit pericarp tissues were harvested at 52 days post anthesis (DPA).

\section{Annotation of LTR retrotransposons}

Functional de novo annotation of LTR retrotransposons for the genomes of TAIR10 (Arabidopsis), MSU7 (rice) and SL2.50 (tomato) was achieved by the LTRpred pipeline (https://github.com/HajkD/LTRpred) using the parameter configuration: minlenltr $=100$, maxlenltr $=5000$, mindistltr $=4000$, maxdisltr $=30000$, mintsd $=3$, maxtsd $=20$, vic $=80$, overlaps $=$ "no", $x$ drop $=7$, motifmis $=1$, pbsradius $=60$, pbsalilen $=c(8,40)$, pbsoffset $=$ $c(0,10)$, quality.filter $=$ TRUE, $n$.orf $=0$. The plant-specific tRNAs used to screen for primer binding sites (PBS) were retrieved from GtRNAdb36 and plantRNA37 and combined in a custom fasta file. The hidden Markov model files for gag and pol protein conservation screening were retrieved from Pfam38 using the protein domains RdRP_1 (PF00680), RdRP_2 (PF00978), RdRP_3 (PF00998), RdRP_4 (PF02123), RVT_1 (PF00078), RVT_2 (PF07727), Integrase DNA binding domain (PF00552), Integrase zinc binding domain (PF02022), Retrotrans_gag (PF03732), RNase H (PF00075) and Integrase core domain (PF00665). Computationally reproducible scripts for generating annotations can be found at http://github.com/HajkD/ALE.

\section{ALE-seq library preparation}

Genomic DNA was extracted using a DNeasy Plant Mini Kit (Qiagen) following the manufacturer's instruction. Genomic DNA (100 ng) was used for adapter ligation with $4 \mu \mathrm{l}$ of $50 \mu \mathrm{M}$ adapter DNA. After an overnight ligation reaction at $4{ }^{\circ} \mathrm{C}$, the adapter-ligated DNA was purified by AMPure XP beads (Beckman Coulter) at a 1:0.5 ratio. In vitro transcription reactions were performed using a MEGAscript RNAi kit (Thermo Fisher) with minor modifications. Briefly, the reaction was carried out for $4 \mathrm{~h}$ at $37^{\circ} \mathrm{C}$ and the template DNA was digested prior to RNA purification. Purified RNA ( $3 \mu \mathrm{g})$ was subjected to reverse transcription (RT) using a Transcriptor First Strand cDNA Synthesis Kit (Roche). Transcriptor First Strand cDNA Synthesis Kit was chosen because the RTase of the kit is thermostable. This allowed the RT reaction at higher temperature $\left(55^{\circ} \mathrm{C}\right)$ that reduces the RT-inhibiting RNA secondary structure formation. The custom RT primers were added as indicated for each experiment. After the RT reaction, $1 \mu \mathrm{l}$ of RNase A/T1 (Thermo Fisher) was added to digest non-templated RNA and the reaction mixture was incubated at $37^{\circ} \mathrm{C}$ for at least $30 \mathrm{~min}$. Single-stranded first strand cDNA was PCR-amplified by 25 cycles using Illumina TruSeq HT dual adapter primers and the PCR product was purified by AMPure XP beads (Beckman Coulter) at a 1:1 ratio. After purification, the eluted DNA was quantified using a KAPA Library Quantification Kit (KAPA Biosystems) and run on the MiSeq v3 2 X 300 bp platform in the Department of Pathology of the University of Cambridge. Due to the nature of ALE-seq that specifically amplifies ecDNAs, some ecDNA-free samples did not produce enough library DNAs which, although suboptimal loading, were nevertheless 
sequenced. It is advisable to spike in PCR-amplified retrotransposon DNA as described below. The oligonucleotide sequences are provided in Supplementary Table 1.

\section{Preparation of full-length Onsen DNA}

The full-length Onsen copy (AT1TE12295) was amplified using Phusion High-Fidelity DNA polymerase (New England Biolabs). PCR products were run on 1\% agarose gels. The fulllength fragment was then purified by QIAquick Gel Extraction (Qiagen) and its concentration was measured using the Qubit Fluorometric Quantitation system (Thermo Fisher). Primers used for amplification are listed in Supplementary Table 1.

\section{RT-qPCR analyses}

Samples were ground in liquid nitrogen using mortar and pestle. An RNeasy Plant Mini Kit (Qiagen) was used to extract total RNA following the manufacturer's instructions. The amount of extracted RNA was estimated using the Qubit Fluorometric Quantitation system (Thermo Fisher). cDNAs were synthesized using a SuperScript VILO cDNA Synthesis Kit (Invitrogen). Real-time quantitative PCR was performed in the LightCycler 480 system (Roche) using primers listed in Supplementary Table 1. LightCycler 480 SYBR green I master premix (Roche) was used to prepare the reaction mixture in a volume of $10 \mu$. The results were analysed by the $\Delta \Delta \mathrm{Ct}$ method.

\section{RNA-seq library construction}

Total RNA was prepared as described above. An Illumina TruSeq Stranded mRNA Library Prep kit (Illumina) was used according to the manufacturer's instructions. The resulting library was run on an Illumina NextSeq 500 machine (Illumina) in the Sainsbury Laboratory at the University of Cambridge.

\section{Analysis of next-generation sequencing data}

For RNA-seq data analysis, the adapter and the low-quality sequences were removed by Trimmomatic software39. The cleaned reads were mapped to the MSU7 version of the rice reference genome (http://rice.plantbiology.msu.edu) using TopHat240. The resulting mapping files were processed to the Cufflinks/Cuffquant/Cuffnorm pipeline41 guided by the annotation file which includes the MSU7 reference gene annotation (http:// rice.plantbiology.msu.edu/pub/data/Eukaryotic_Projects/o_sativa/annotation_dbs/ pseudomolecules/version_7.0/all.dir/) and our custom retrotransposon annotation. Visualization of sequencing data was performed using an Integrative Genomics Viewer (IGV)42.

For ALE-seq data analysis, the adapter sequence was removed from the raw reads using Trimmomatic software. For the mapping-based approach, paired-end reads were mapped to the reference genomes (Arabidopsis, TAIR10; rice, MSU7; tomato, SL2.50) using Bowtie243 with minor optimization. In most short-read sequencing platforms, it is often difficult to assign the multi-mapped reads of TEs to precise genomic location. However, as MiSeq outputs relatively longer reads, we presumed that ALE-seq reads have less ambiguity than other sequencing platforms and set the parameters dealing with multi-mappers to default. It is only the maximum fragment length option which is set to 500 by default that 
was manipulated to 3000 (-X 3000). The numbers of reads mapped throughout each retrotransposon were counted by the featureCounts tool of the SubRead package44 using the custom annotation file created by LTRpred. Since featureCounts recognizes multi-mappers by SAM file's NH tag that bowtie2 does not generate, multi-mapped reads are counted as one read aligned to a single genomic location, which reduces quantitation bias that often happens to multi-mappers. IGV was used to visualize the sequencing data. For the alignment-based approach, the forward and reverse reads were merged to yield the fulllength fragment sequences and converted to fasta files using the BBTools (https:// jgi.doe.gov/data-and-tools/bbtools/). The fasta files created for all the samples were concatenated to get a master fasta file that is later inputted to CD-HIT software 45 to cluster the reads by sequence similarity with the following options: -c 0.95 , -ap 1, -g 1. CD-HIT outputs a fasta file of representative reads for each cluster. The resulting fasta file was used as reference for paired-end mapping of initial fastq files. The mapped reads were counted with the featureCounts tool. Those clusters that significantly differed in the number of mapped reads in different samples were further analysed for their identities using BLAST search.

For Bisulfite sequencing analysis, raw sequenced reads derived from tomato fruits (52 DPA) and leaves were downloaded from the public repository (SRP008329)16 and re-analysed as previously described46, with minor modifications. Briefly, high-quality sequenced reads were mapped with Bismark47 on the cv. Heinz 1706 reference genome (https:// solgenomics.net), including a chloroplast sequence obtained from GenBank database (NC_007898.3) to estimate the conversion rate. After methylation call and correction for unconverted cytosines, the methylation proportions at each cytosine position with a coverage of at least 3 reads were used to generate a bedGraph file for each cytosine context, using the R Bioconductor packages DMRCaller48 and Rtracklayer49. The IGV browser was used to visualize the methylation profiles.

\section{Detection of retrotransposon insertions}

The insertions of selected retrotransposons were detected from the genome resequencing data of japonica and indica rice accessions downloaded from the 3,000 rice genome project (PRJEB6180). The Transposon Insertion Finder (TIF) program14 was used to identify the split reads in the fastq files and detect newly integrated copies. We used MSU7 (http:// rice.plantbiology.msu.edu) and ShuHui498 (http://www.mbkbase.org) for the reference of japonica and indica rice, respectively. Only non-reference insertions were considered and common insertions found in multiple accessions were counted as a single retrotransposition event.

\section{Supplementary Material}

Refer to Web version on PubMed Central for supplementary material.

\section{Acknowledgements}

This work was supported by European Research Council (EVOBREED) [322621]; Gatsby Fellowship [AT3273/ GLE]. 


\section{References}

1. Lisch D. How important are transposons for plant evolution? Nat Rev Genet. 2012; 14:49-61.

2. Chuong EB, Elde NC, Feschotte C. Regulatory activities of transposable elements: from conflicts to benefits. Nat Rev Genet. 2017; 18:71-86. [PubMed: 27867194]

3. Griffiths J, Catoni M, Iwasaki M, Paszkowski J. Sequence-Independent Identification of Active LTR Retrotransposons in Arabidopsis. Mol Plant. 2017; 11:508-511. [PubMed: 29107035]

4. Ma J, Bennetzen JL. Rapid recent growth and divergence of rice nuclear genomes. Proc Natl Acad Sci U S A. 2004; 101:12404-12410. [PubMed: 15240870]

5. Sanchez DH, Gaubert H, Drost H, Zabet NR, Paszkowski J. High-frequency recombination between members of an LTR retrotransposon family during transposition bursts. Nat Commun. 2017; 8:1-6. [PubMed: 28232747]

6. Picault N, et al. Identification of an active LTR retrotransposon in rice. Plant J. 2009; 58:754-765. [PubMed: 19187041]

7. Sabot F, et al. Transpositional landscape of the rice genome revealed by paired-end mapping of high-throughput re-sequencing data. Plant J. 2011; 66:241-246. [PubMed: 21219509]

8. Mirouze M, et al. Selective epigenetic control of retrotransposition in Arabidopsis. Nature. 2009; 461:427-430. [PubMed: 19734882]

9. Ito $\mathrm{H}$, et al. An siRNA pathway prevents transgenerational retrotransposition in plants subjected to stress. Nature. 2011; 472:115-119. [PubMed: 21399627]

10. Paszkowski J. Controlled activation of retrotransposition for plant breeding. Curr Opin Biotechnol. 2015; 32:200-206. [PubMed: 25615932]

11. Cavrak VV, et al. How a Retrotransposon Exploits the Plant's Heat Stress Response for Its Activation. PLoS Genet. 2014; 10:1-12.

12. Pietzenuk B, et al. Recurrent evolution of heat-responsiveness in Brassicaceae COPIA elements. Genome Biol. 2016; :1-15. DOI: 10.1186/s13059-016-1072-3 [PubMed: 26753840]

13. The 3, 000 rice genomes project. The 3, 000 rice genomes project. Gigascience. $2014 ; 3: 1-6$. [PubMed: 24460651]

14. Nakagome M, et al. Transposon Insertion Finder (TIF): a novel program for detection of de novo transpositions of transposable elements. BMC Bioinformatics. 2014; 15:1-9. [PubMed: 24383880]

15. Xiong ZY, et al. Latitudinal Distribution and Differentiation of Rice Germplasm: Its Implications in Breeding. Crop Sci. 2011; 51:1050-1058.

16. Zhong S, et al. Single-base resolution methylomes of tomato fruit development reveal epigenome modifications associated with ripening. Nat Biotechnol. 2013; 31:154-159. [PubMed: 23354102]

17. Consortium $\mathrm{T}$. tomato genome. The tomato genome sequence provides insights into fleshy fruit evolution. Nature. 2012; 485:635-641. [PubMed: 22660326]

18. Eshed Y, Zamir D. An Introgression Line Population of Lycopersicon pennellii in the Cultivated Tomato Enables the Identification and Fine Mapping of Yield-Associated QTL. Genetics. 1995; 141:1147-1162. [PubMed: 8582620]

19. Eshed Y, Zamir D. Less-Than-Additive Epistatic Interactions of Quantitative Trait Loci in Tomato. Genetics. 1996; 143:1807-1817. [PubMed: 8844166]

20. Quadrana L, et al. The Arabidopsis thaliana mobilome and its impact at the species level. Elife. 2016; 5:1-25.

21. Stuart T, et al. Population scale mapping of transposable element diversity reveals links to gene regulation and epigenomic variation. Elife. 2016; 5:1-27.

22. Wei B, et al. Genome-wide characterization of non-reference transposons in crops suggests nonrandom insertion. BMC Genomics. 2016; 17:1-13. [PubMed: 26818753]

23. Lanciano S, et al. Sequencing the extrachromosomal circular mobilome reveals retrotransposon activity in plants. PLOS Genetics. 2017; 13

24. Møller HD, et al. Formation of Extrachromosomal Circular DNA from Long Terminal Repeats of Retrotransposons in Saccharomyces cerevisiae. G3 (Bethesda). 2015; 6:453-462. [PubMed: 26681518] 
25. Møller HD, Parsons L, Jørgensen TS, Botstein D, Regenberg B. Extrachromosomal circular DNA is common in yeast. Proc Natl Acad Sci U S A. 2015; 112:3114-3122.

26. Cheng $\mathrm{C}$, et al. Loss of function mutations in the rice chromomethylase OsCMT3a cause a burst of transposition. Plant J. 2015; 83:1069-1081. [PubMed: 26243209]

27. Cui X, et al. Control of transposon activity by a histone H3K4 demethylase in rice. Proc Natl Acad Sci U S A. 2013; 110:1953-1958. [PubMed: 23319643]

28. Wang ZH, et al. Genomewide Variation in an Introgression Line of Rice-Zizania Revealed by Whole-Genome re-Sequencing. PLoS One. 2013; 8:1-12.

29. Li H, Freeling M, Lisch D. Epigenetic reprogramming during vegetative phase change in maize. Proc Natl Acad Sci U S A. 2010; 107:22184-22189. [PubMed: 21135217]

30. Slotkin RK, et al. Epigenetic Reprogramming and Small RNA Silencing of Transposable Elements in Pollen. Cell. 2009; 136:461-472. [PubMed: 19203581]

31. Liu R, et al. A DEMETER-like DNA demethylase governs tomato fruit ripening. Proc Natl Acad Sci. 2015; 112:10804-10809. [PubMed: 26261318]

32. Goodier JL. Retrotransposition in tumors and brains. Mobile DNA. 2014; 5:1-6. [PubMed: 24382139]

33. Baillie JK, et al. Somatic retrotransposition alters the genetic landscape of the human brain. Nature. 2011; 479:534-537. [PubMed: 22037309]

34. Mullins CS, Linnebacher M. Human endogenous retroviruses and cancer : Causality and therapeutic possibilities. World J Gastroenterol. 2012; 18:6027-6035. [PubMed: 23155332]

35. Cho J, Paszkowski J. Regulation of rice root development by a retrotransposon acting as a microRNA sponge. Elife. 2017; 6:1-21.

36. Chan PP, Lowe TM. GtRNAdb 2 . 0 : an expanded database of transfer RNA genes identified in complete and draft genomes. Nucleic Acids Res. 2016; 44:184-189.

37. Daujat M, et al. PlantRNA, a database for tRNAs of photosynthetic eukaryotes. Nucleic Acids Res. 2012; 41:273-279.

38. Finn RD, et al. Pfam : the protein families database. Nucleic Acids Res. 2014; 42:222-230.

39. Bolger AM, Lohse M, Usadel B. Trimmomatic : a flexible trimmer for Illumina sequence data. Bioinformatics. 2014; 30:2114-2120. [PubMed: 24695404]

40. Kim D, et al. TopHat 2 : accurate alignment of transcriptomes in the presence of insertions, deletions and gene fusions. Genome Biol. 2013; 14:1-13.

41. Trapnell C, et al. Transcript assembly and quantification by RNA-Seq reveals unannotated transcripts and isoform switching during cell differentiation. Nat Biotechnol. 2010; 28:516-520. [PubMed: 20436463]

42. Robinson JT, et al. Integrative Genomics Viewer. Nat Biotechnol. 2011; 29:24-26. [PubMed: 21221095]

43. Langmead B, Salzberg SL. Fast gapped-read alignment with Bowtie 2. Nat Methods. 2013; 9:357359.

44. Liao Y, Smyth GK, Shi W. The Subread aligner : fast, accurate and scalable read mapping by seedand-vote. Nucleic Acids Res. 2013; 41:1-17. [PubMed: 23143271]

45. Li W, Godzik A. Cd-hit : a fast program for clustering and comparing large sets of protein or nucleotide sequences. Bioinformatics. 2006; 22:1658-1659. [PubMed: 16731699]

46. Catoni M, et al. DNA sequence properties that predict susceptibility to epiallelic switching. EMBO. 2017; 36:617-628.

47. Krueger F, Andrews SR. Bismark : a flexible aligner and methylation caller for Bisulfite-Seq applications. Bioinformatics. 2011; 27:1571-1572. [PubMed: 21493656]

48. Catoni M, Tsang JMF, Greco AP, Zabet NR. DMRcaller : a versatile R / Bioconductor package for detection and visualization of differentially methylated regions in $\mathrm{CpG}$ and non-CpG contexts. Nucleic Acids Res. 2018:1-11. [PubMed: 29177436]

49. Lawrence M, Gentleman R, Carey V. rtracklayer : an R package for interfacing with genome browsers. Bioinformatics. 2009; 25:1841-1842. [PubMed: 19468054] 
a

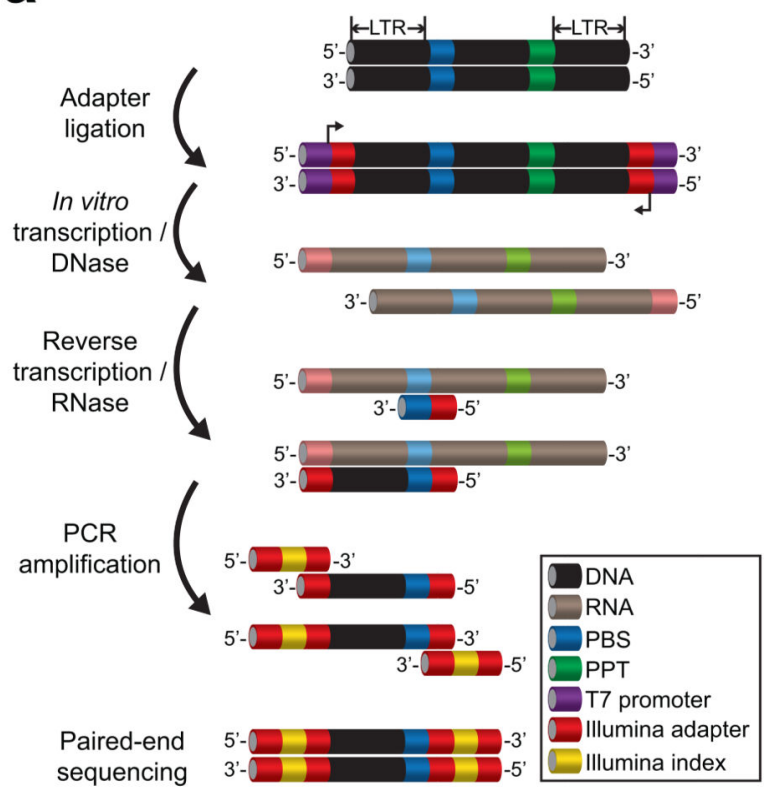

C

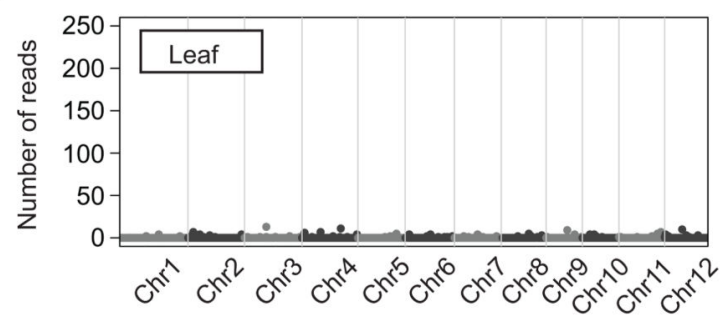

e

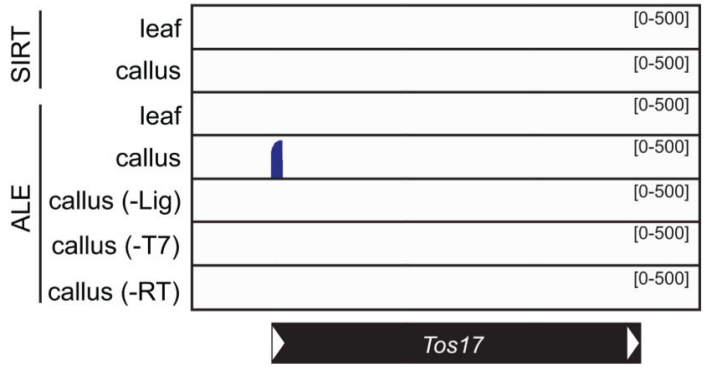

b

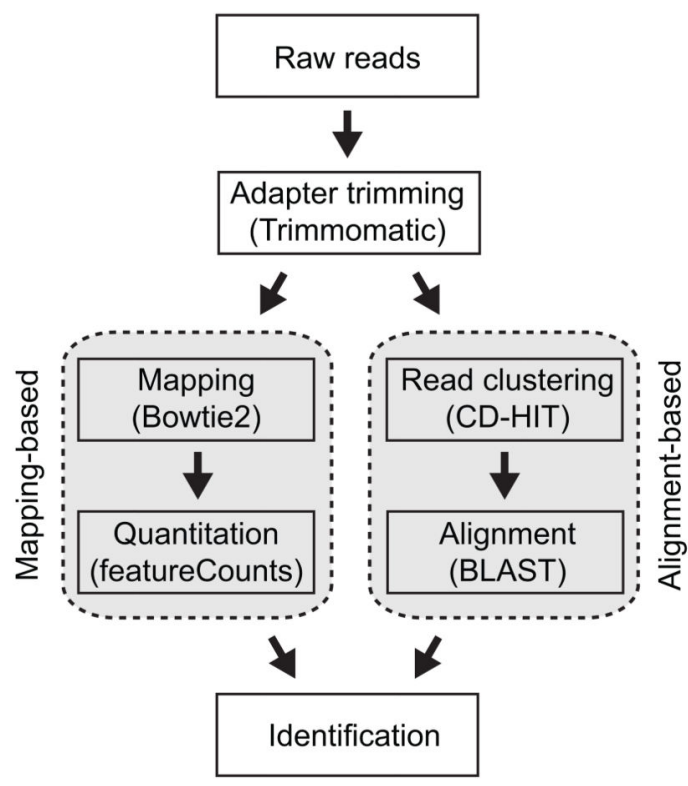

d

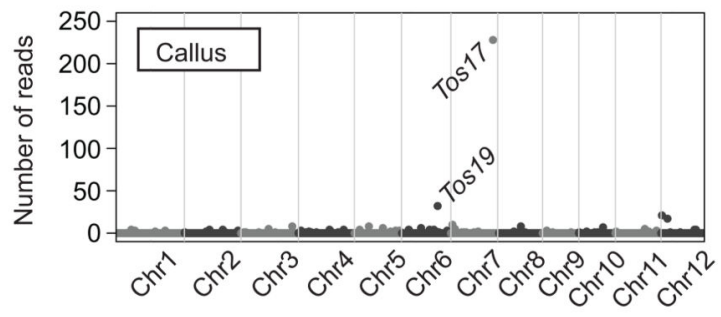

f

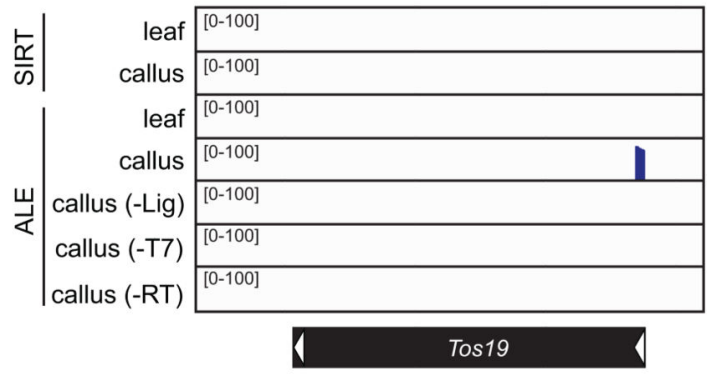

Figure 1. Detection of ecIDNA by ALE-seq

a, The workflow of ALE-seq. The colour code is indicated in a box. $\mathbf{b}$, Analysis pipeline of ALE-seq results. The sequenced reads can be mapped to the reference genome or aligned to each other to obtain a cluster consensus. $\mathbf{c}$ and $\mathbf{d}$, Genome-wide plots of rice ALE-seq results from leaf (c) and callus (d). The levels are shown as number of reads mapped to each retrotransposon. Dots represent annotated retrotransposons; those corresponding to Tos 17 and $\operatorname{Tos} 19$ are indicated. e and f, Read coverage plots mapped to $\operatorname{Tos} 17(\mathbf{e})$ and $\operatorname{Tos} 19(\mathbf{f})$. The black bars represent retrotransposons and white arrowheads indicate LTRs. 
a

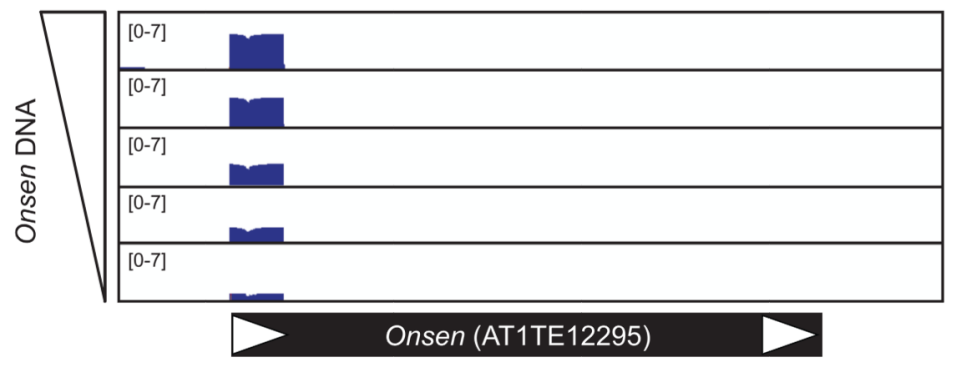

C

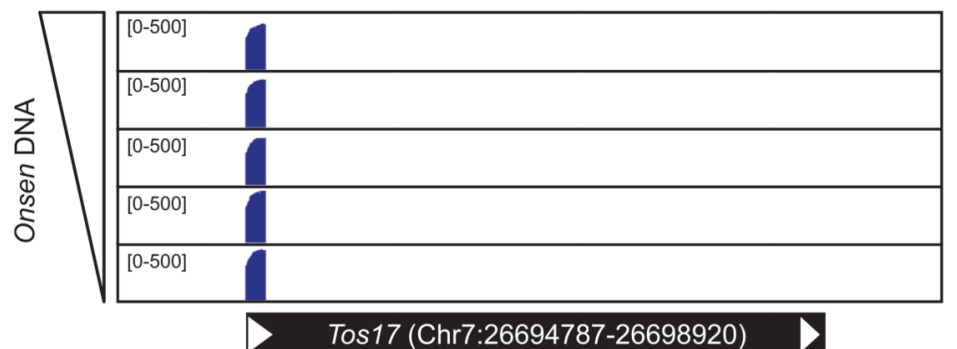

b

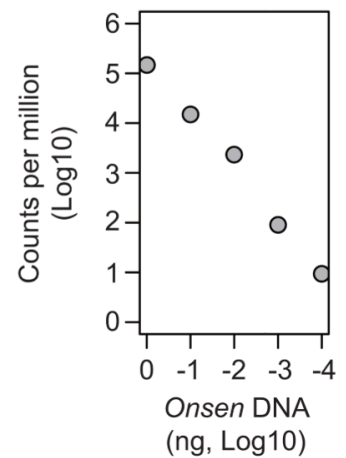

d

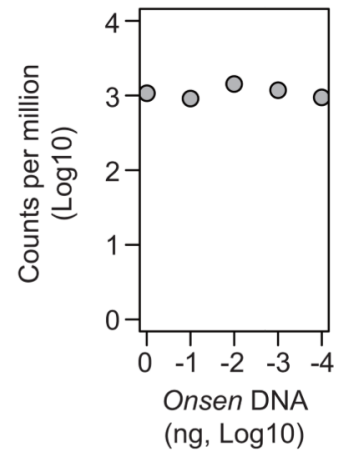

$\mathbf{e}$

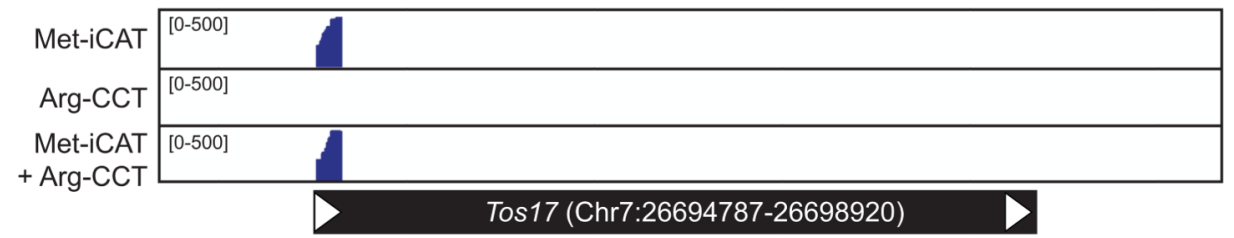

\section{f}

\begin{tabular}{r|l|l|} 
Met-iCAT & {$[0-100]$} & \\
Arg-CCT & {$[0-100]$} & \\
Met-iCAT & {$[0-100]$} & \\
+ +Arg-CCT & & \\
\cline { 2 - 2 } & &
\end{tabular}

Figure 2. Sensitivity and specificity of ecIDNA detection by ALE-seq

a-d, ALE-seq reconstruction experiment with varying amounts of PCR-amplified Onsen DNA added to rice callus DNA. Genome browser image with the read coverage (a and c) and quantitated read counts (b and $\mathbf{d}$ ) for Onsen (a and b) and Tos17(c and d) loci. The amounts of Onsen DNA added were $1 \mathrm{ng}, 100 \mathrm{pg}, 10 \mathrm{pg}, 1 \mathrm{pg}$ or $100 \mathrm{fg} ; 100 \mathrm{ng}$ of rice callus DNA was used. Note that read coverage values are $\log _{10}$-converted in $\mathbf{a}$. For $\mathbf{b}$ and $\mathbf{d}$, values are shown as $\log _{10}$-converted counts per million sequenced reads. e and $\mathbf{f}$, Read 
coverage plots for the ALE-seq of rice callus using different RT primers. Tos 17 and RIRE2 transposons are depicted below the plots as in Figure 1. 
a

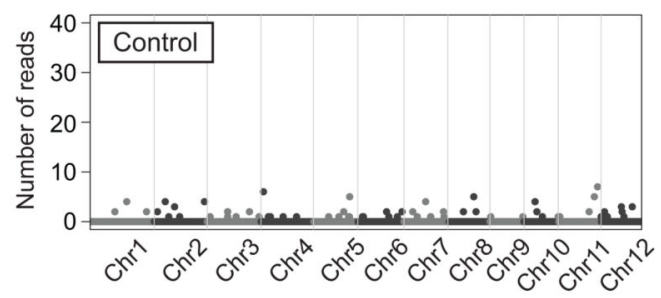

b

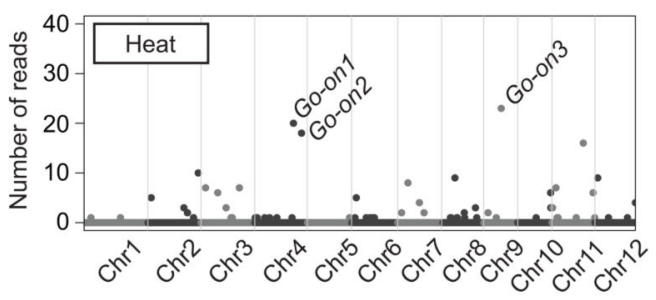

C

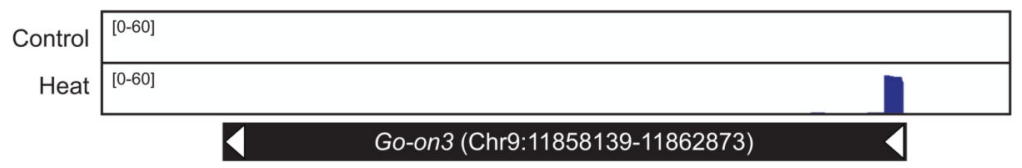

d

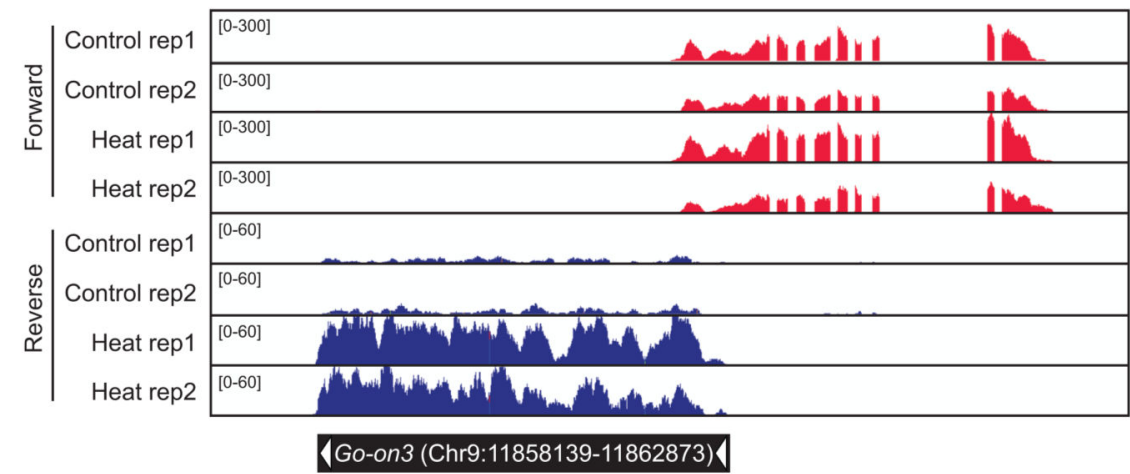

LOC_Os09g19820

e

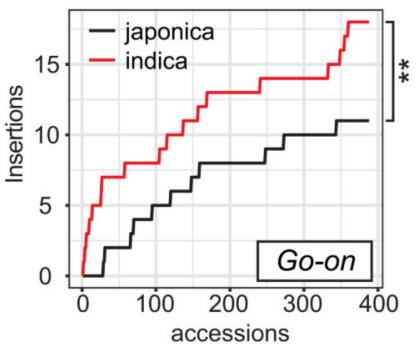

f

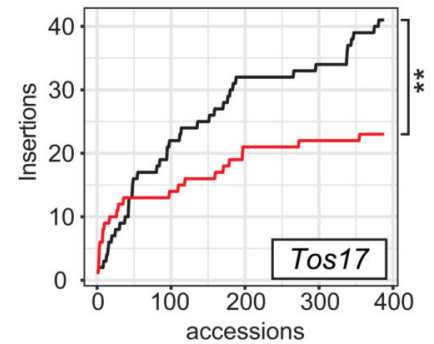

g

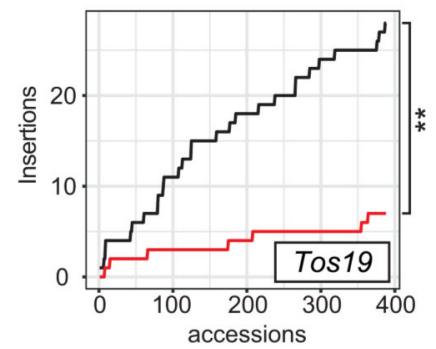

Figure 3. Identification of a novel heat-activated retrotransposon in rice $\mathbf{a}$ and $\mathbf{b}$, Genome-wide plots of rice ALE-seq results as in Figure 1. Control (a) and heatstressed (b) rice plants were used. One-week-old seedlings were subjected to heat stress $\left(44^{\circ} \mathrm{C}\right)$ for 3 days. Met-iCAT PBS primer was used in RT. The levels are shown as the number of reads mapped to retroelements. Three Go-on copies are indicated in b. c, Read coverage plot for Go-on3. d, RNA-seq data showing Go-on3 and a neighbouring gene. RNA-seq data were generated using the same plant materials as in $\mathbf{a}$ and $\mathbf{b}$. The experiment was repeated independently two times with similar results. e-g, Cumulative plots for the 
number of non-reference insertions of Go-on (e), $\operatorname{Tos} 17(\mathbf{f})$, and $\operatorname{Tos} 19(\mathbf{g})$ in the genomes of 388 japonica and indica rice accessions. The statistical difference was determined by iterating random selection of 200 accessions out of 388 and performing the two-tailed Wilcoxon test. $* * P=2.2 \mathrm{e}^{-16}$. 
a

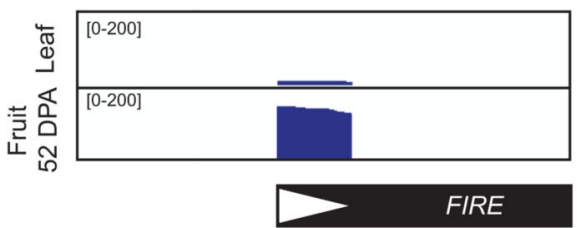

b

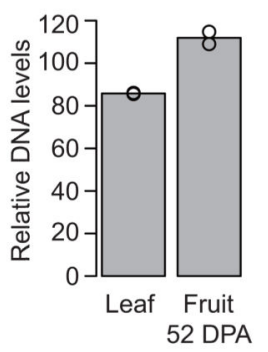

C

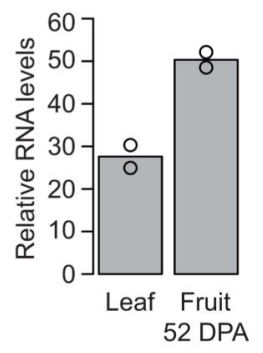

d

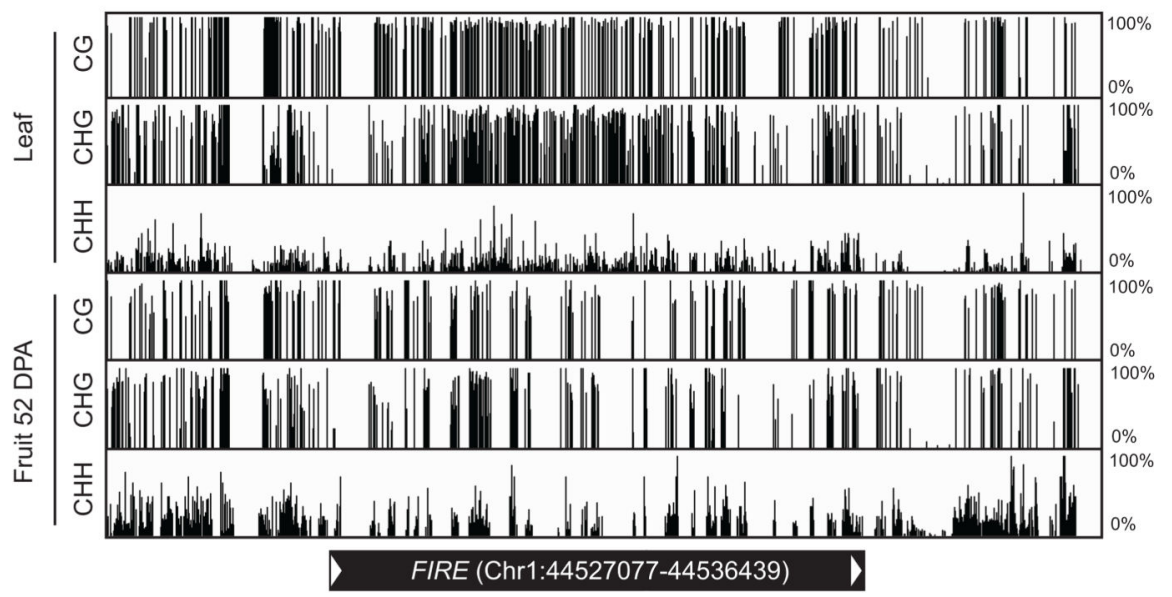

e

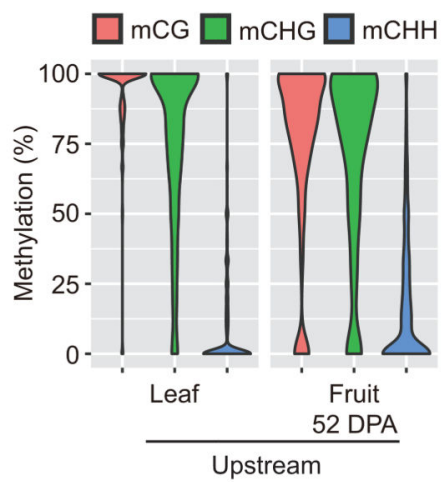

$\mathbf{f}$

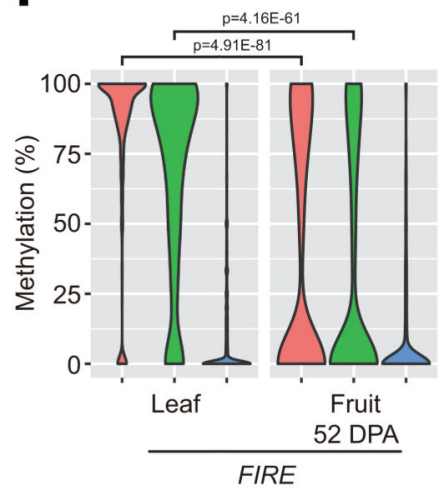

g

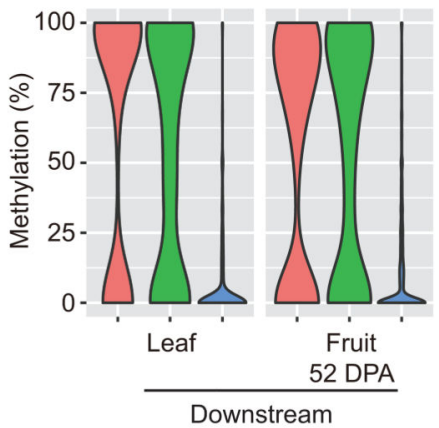

Figure 4. Identification of a tomato retrotransposon activated in fruit pericarp

a, Read coverage plot for the FIRE retrotransposon identified in tomato fruit pericarp by ALE-seq. Met-iCAT PBS primer was used in RT. b and $\mathbf{c}$, The DNA (b) and RNA (c) levels of FIRE in leaves and fruits determined by qPCR. The levels are means of two biological replicates. Normalization was done against SIGAPDH(Solyc03g111010) and SICAC (Solyc08g006960) for DNA and RNA analyses, respectively. d, Genome browser image for the DNA methylation levels at FIRE element in leaves and fruits of tomato. The levels are shown as percent methylation of each cytosine. $\mathbf{e - g}$, Violin plots for DNA methylation levels 
at the upstream (e), $\operatorname{FIRE}(\mathbf{f})$ and downstream (g) regions. Only cytosines supported by at least three reads in both samples were considered. In FIRE locus, for example, 4,032 out of 4,078 cytosines in both strands were analysed. The upstream and downstream regions are immediate flanking sequences taken for the same length as FIRE of $9.362 \mathrm{~kb}$. P-values were determined by a two-sided Fisher's t-test using $558 \mathrm{CG}$ and 717 CHG sites at FIRE locus. Other samples with insignificant statistical difference are not shown for the p-values. 